\title{
Association Between Non-Cystic Fibrosis Bronchiectasis and Quality of Life: A Single- Center Cross-Sectional Study
}

\author{
Sheetal Chaurasia ${ }^{1}$, Alamelu Haran ${ }^{2}$, Anish Reddy ${ }^{2}$, Pavny Chawla ${ }^{3}$ \\ 1. Pulmonary Medicine, Manipal Hospitals Whitefield, Bangalore, IND 2. Pulmonary Medicine, Vydehi Institute of \\ Medical Sciences and Research Center, Bangalore, IND 3. Respiratory Diseases and Sleep Disorders, Artemis Hospital, \\ Gurgaon, IND
}

Corresponding author: Sheetal Chaurasia, sheetal.utkalit@gmail.com

\section{Abstract}

\section{Introduction}

Bronchiectasis is a chronic respiratory disease that can affect patients of all ages and significantly impact the quality of life (QOL) in patients who suffer from it. In spite of its widespread prevalence, and the significant impact on QOL, data on the quantitative impact of bronchiectasis on QOL is lacking. The Quality of Life-Bronchiectasis (QOL-B) is a self-administered patient-reported outcome measure, that was recently developed as a response to the emergent need for such measurement tools to study the impact of bronchiectasis on QOL.

\section{Methods}

We conducted a single-center cross-sectional study to study the correlation between QOL and various other outcome parameters such as exercise capacity, lung functions, co-morbidities, inflammatory markers, and body mass index (BMI). The secondary outcome was to find out various determinants of quality of life in non-cystic fibrosis bronchiectasis (NCFB).

\section{Results}

Forty-four patients who determined the pre-determined criteria for NCFB were enrolled in this study. This study demonstrated a significant impact on the QOL of NCFB patients based on the QOL-B scoring system. Almost all domains of QOL-B were found to be adversely impacted as measured by one or more of the outcome parameters but the FEV1, age, colonization, extension, dyspnea (FACED) score, bronchiectasis severity index (BSI) score, six-minute walk test (6MWD), and FEV1 showed associations across most scales while the other outcome parameters showed varying associations.

\section{Conclusions}

The QOL is significantly reduced in NCFB and it may be quantified using the QOL-B questionnaire. The

Review began 03/17/2021 Review ended 03/25/2021 Published 03/31/2021

๑) Copyright 2021

Chaurasia et al. This is an open access article distributed under the terms of the Creative Commons Attribution License CC-BY 4.0., which permits unrestricted use, distribution, and reproduction in any medium, provided the original author and source are credited. impact on QOL in NCFB may be assessed using validated tools such as the FACED and BSI scoring systems, as well as other well-established outcome parameters like 6MWD and FEV1 predicted.

Categories: Pulmonology

Keywords: bronchiectasis, quality of life, exercise capacity

\section{Introduction}

Bronchiectasis is a chronic respiratory disease that can affect patients of all ages. It is more prevalent in women as the age advances, and there is an increasing trend in the prevalence recently [1]. The underlying etiology in bronchiectasis could involve cystic fibrosis (CF), alpha-1-antitrypsin deficiency, primary ciliary dyskinesia, allergic bronchopulmonary aspergillosis, connective tissue disorders, inflammatory bowel diseases, congenital malformations, aspiration, humoral immunodeficiency, post-infectious, and finally idiopathic cases [2]. The pathophysiology behind bronchiectasis includes chronic inflammatory microenvironments that can trigger the breakdown of the airway tissue. The mechanism remains more or less the same in both CF and non-CF bronchiectasis, with a complex interplay between infection and inflammation that feeds a pro-inflammatory vicious cycle. This cycle progressively drives the formation of bronchiectasis and subsequent destruction of the pulmonary architecture [3].

Patients generally present with daily excessive sputum and other associated symptoms, recurrent chest infections and as a consequence of the chronic course with recurrent exacerbations, am impaired healthrelated quality of life (QOL) [2]. In spite of its widespread prevalence, and the significant impact on QOL, data on the quantitative impact of bronchiectasis on QOL is lacking. The Quality of Life-Bronchiectasis (QOL-B) is a self-administered patient-reported outcome measure, that was recently developed as 
a response to the emergent need for such measurement tools to study the impact of bronchiectasis on QOL. It is useful in the assessment of the symptoms, functioning, and health-related quality of life for non-CF bronchiectasis patients. It includes 37 parameters on eight scales including respiratory symptoms, physical, role, emotional, and social functioning, vitality, health perceptions, and treatment burden [4].

In this study, we looked at the various determinants of QOL in non-cystic fibrosis bronchiectasis (NCFB) and the correlation of QOL with clinical, radiological, and physiological parameters in bronchiectasis.

\section{Materials And Methods}

This study was conducted as a single-center cross-sectional study in a 1000 bedded tertiary care hospital in the state of Karnataka, India between January 2016 and December 2016. Forty-four consecutive adult patients above the age of 18 years, who had NCFB and were clinically stable were included in this study after informed consent. The exclusion criteria consisted of other co-existent respiratory disorders or chronic debilitating disorders involving any system which could confound results, pregnant women and children, and patients who were not willing to participate in the study.

The primary objective of this study was to study the correlation between QOL and various other outcome parameters like exercise capacity, lung functions, co-morbidities, inflammatory markers, and BMI. The secondary outcome was to find out various determinants of quality of life in NCFB.

The diagnosis of NCFB was confirmed by high-resolution computerized tomography (HRCT) thorax. After a detailed history and relevant investigations, the following outcome parameters were obtained: Modified Medical Research Council (MMRC) grading, body mass index (BMI), six-minute walking distance (6MWD), Creactive protein (CRP), and the forced expiratory volume in one second (FEV1). The severity of NCFB was stratified using two validated scores: the FEV1, age, colonization, extension, dyspnea (FACED) score and the bronchiectasis severity index (BSI) [2]. The FACED score is used to predict the risk of five-year mortality. It is calculated using FEV1\% predicted, age, chronic Pseudomonas colonization, the extent of bronchiectasis, and the Medical Research Council Dyspnea Scale score [2]. The BSI was calculated using an online tool. The determinants of BSI include age, body mass index, FEV1\% predicted, Medical Research Council Dyspnea Scale score, lobes affected, and evidence of chronic bacterial infection [2].

The QOL was assessed using the QOL-B questionnaire (Version 3.0). The QOL-B questionnaire is a selfreported questionnaire consisting of 37 items based on eight scales (respiratory symptoms $\{R S\}$, physical $\{\mathrm{P}\}$, role $\{R\}$, emotional $\{\mathrm{E}\}$, social functioning $\{\mathrm{S}\}$, vitality $\{\mathrm{V}\}$, health perceptions $\{\mathrm{HP}\}$, and treatment burden \{T\}) [4]. Data were collected on the number of exacerbations, the use of antibiotics, oral steroids, hospitalizations, or emergency department admissions in the previous month. The outcome parameters were correlated with each scale of QOL- B separately.

Statistical analysis was done by STATA Version 11.2 (StataCorp, College Station, TX). Descriptive statistics were used to compute baseline data according to data distribution. Mean differences were compared using ttest or analysis of variance (ANOVA) as applicable and median using Kruskal Wallis test. Correlation between different parameters was done by Pearson or Spearman correlation coefficient depending on the distribution of the values. Predictors of different domains of quality of life were found out by multivariate regression analysis.

Even though it was a questionnaire-based study, an ethical committee clearance was obtained for this study, and all patients were enrolled after informed consent. The study is reported in accordance with the Strengthening the Reporting of Observational Studies in Epidemiology (STROBE) statement [5].

\section{Results}

Forty-four patients who determined the pre-determined criteria for NCFB were enrolled in this study. There were $24(55 \%)$ males and $20(45 \%)$ females with a mean age of $43.4 \pm 11.7$ years. There were four patients (9\%) in the MMRC grade zero category, eight patients (18\%) in the MMRC grade one category, 13 patients (30\%) in the MMRC grade two category, 15 patients (34\%) in the MMRC grade three category, and four patients (9\%) in the MMRC grade four category. Comparison between QOL-B scales and MMRC grades showed significant associations between higher MMRC grades and poorer scores in respiratory symptoms, physical, role, emotional, social functioning, vitality, health perceptions, and treatment burden scales (Table 1). 


\section{Cureus}

\begin{tabular}{|c|c|c|c|c|c|c|}
\hline & \multicolumn{5}{|l|}{ MMRC grade } & \multirow[b]{2}{*}{$\mathrm{p}$-Value } \\
\hline & 0 & 1 & 2 & 3 & 4 & \\
\hline & Mean \pm SD & Mean \pm SD & Mean \pm SD & Mean \pm SD & Mean \pm SD & \\
\hline Physical & $53.75 \pm 20.20$ & $67.45 \pm 21.46$ & $49.72 \pm 20.96$ & $30.11 \pm 23.09$ & $6.67 \pm 0$ & $<0.001$ \\
\hline Role & $60.01 \pm 19.60$ & $62.46 \pm 15.48$ & $54.36 \pm 29.29$ & $43.55 \pm 18.49$ & $33.33 \pm 9.43$ & 0.125 \\
\hline Vitality & $63.84 \pm 16.63$ & $53.88 \pm 23.05$ & $54.33 \pm 20.06$ & $36.29 \pm 19.03$ & $22.83 \pm 16.29$ & 0.008 \\
\hline Emotional & $70.84 \pm 22.05$ & $77.0 \pm 16.44$ & $66.61 \pm 19.17$ & $57.22 \pm 23.33$ & $31.25 \pm 7.98$ & 0.008 \\
\hline Social & $66.68 \pm 29.66$ & $64.58 \pm 19.79$ & $64.74 \pm 27.24$ & $54.44 \pm 19.89$ & $30.83 \pm 17.92$ & 0.106 \\
\hline Treatment burden & $32.22 \pm 49.32$ & $75.95 \pm 20.45$ & $64.15 \pm 27.14$ & $62.18 \pm 20.11$ & $69.42 \pm 10.53$ & 0.172 \\
\hline Respiratory symptoms & $91.42 \pm 7.68$ & $70.67 \pm 19.22$ & $60.49 \pm 17.68$ & $49.24 \pm 20.83$ & $23.17 \pm 18.77$ & $<0.001$ \\
\hline Health perceptions & $68.75 \pm 24.88$ & $52.21 \pm 20.08$ & $51.66 \pm 20.68$ & $44.53 \pm 21.74$ & $39.50 \pm 14.43$ & 0.234 \\
\hline
\end{tabular}

\section{TABLE 1: Quality of life compared with MMRC}

MMRC: Modified Medical Research Council

Sixteen patients (36\%) had a mild FACED score, eight patients (18\%) had a moderate FACED score, and 20 patients (45\%) had a severe FACED score. Comparison between QOL-B scales and FACED scores showed significant associations between higher FACED score grades and poorer scores in respiratory symptoms, physical, role, emotional, social functioning, vitality, health perceptions, and treatment burden scales (Table 2). Twelve patients (28\%) had a low BSI score, nine patients (20\%) had an intermediate BSI score, and 23 patients (52\%) had a high BSI score. Comparison between QOL-B scales and BSI scores showed significant associations between higher BSI score grades and poorer scores in respiratory symptoms, physical, role, emotional, social functioning, vitality, health perceptions, and treatment burden scales (Table 3).

\begin{tabular}{|c|c|c|c|c|}
\hline & \multicolumn{3}{|l|}{ FACED score } & \multirow[b]{2}{*}{$\mathrm{p}$-Value } \\
\hline & Mild & Moderate & Severe & \\
\hline & Mean \pm SD & Mean \pm SD & Mean \pm SD & \\
\hline Physical & $58.0 \pm 21.09$ & $59.13 \pm 20.53$ & $23.92 \pm 20.78$ & $<0.001$ \\
\hline Role & $61.25 \pm 25.08$ & $59.96 \pm 19.81$ & $38.67 \pm 14.92$ & 0.003 \\
\hline Vitality & $59.25 \pm 17.17$ & $52.79 \pm 23.57$ & $32.89 \pm 18.59$ & 0.001 \\
\hline Emotional & $73.92 \pm 20.12$ & $65.54 \pm 20.98$ & $52.08 \pm 21.27$ & 0.011 \\
\hline Social & $66.15 \pm 27.80$ & $70.83 \pm 13.36$ & $46.99 \pm 19.91$ & 0.013 \\
\hline Treatment burden & $58.69 \pm 34.80$ & $66.67 \pm 19.98$ & $65.38 \pm 18.66$ & 0.728 \\
\hline Respiratory symptoms & $72.39 \pm 17.99$ & $65.87 \pm 15.91$ & $42.45 \pm 22.84$ & $<0.001$ \\
\hline Health perceptions & $56.56 \pm 21.35$ & $54.28 \pm 22.52$ & $42.15 \pm 19.41$ & 0.103 \\
\hline
\end{tabular}

\section{TABLE 2: Quality of life compared with a grade of FACED score}

FACED: FEV1, age, colonization, extension, dyspnea 


\section{Cureus}

\begin{tabular}{|c|c|c|c|c|}
\hline & \multicolumn{3}{|l|}{ BSI score } & \multirow[b]{2}{*}{ p-Value } \\
\hline & Low & Intermediate & High & \\
\hline & Mean \pm SD & Mean \pm SD & Mean \pm SD & \\
\hline Physical & $57.91 \pm 19.68$ & $51.78 \pm 34.34$ & $31.23 \pm 22.97$ & 0.008 \\
\hline Role & $61.67 \pm 22.75$ & $59.22 \pm 21.43$ & $41.74 \pm 19.61$ & 0.017 \\
\hline Vitality & $57.19 \pm 19.20$ & $55.26 \pm 21.42$ & $36.72 \pm 20.77$ & 0.011 \\
\hline Emotional & $72.22 \pm 20.82$ & $73.93 \pm 22.86$ & $52.89 \pm 19.88$ & 0.010 \\
\hline Social & $68.75 \pm 21.81$ & $53.52 \pm 20.50$ & $54.71 \pm 20.07$ & 0.216 \\
\hline Treatment burden & $56.30 \pm 31.82$ & $73.66 \pm 31.98$ & $62.51 \pm 17.07$ & 0.352 \\
\hline Respiratory symptoms & $79.50 \pm 15.28$ & $57.19 \pm 25.43$ & $49.96 \pm 20.79$ & $<0.001$ \\
\hline Health perceptions & $54.95 \pm 24.62$ & $61.67 \pm 14.06$ & $42.08 \pm 19.55$ & 0.035 \\
\hline
\end{tabular}

\section{TABLE 3: Quality of life compared with BSI grade}

BSI: bronchiectasis severity index

In the physical scale of QOL-B, multivariate regression analysis for predictors of low score showed associations with 6MWD and BMI. There were no associations with MMRC, CRP, FEV1, colonizations, FACED scores, and BSI scores (Table 4).

\begin{tabular}{|c|c|c|c|c|c|c|}
\hline & \multicolumn{3}{|c|}{ Simple linear regression } & \multicolumn{3}{|c|}{ Multiple linear regression } \\
\hline & $\beta$ coefficient & $95 \% \mathrm{Cl}$ & $\mathrm{p}$-Value & $\beta$ coefficient & $95 \% \mathrm{Cl}$ & $\mathrm{p}$-Value \\
\hline MMRC & -14.17 & -20.16 to -8.17 & $<0.001$ & 1.75 & -8.21 to 11.71 & 0.723 \\
\hline 6-minute walk test & 0.17 & 0.11 to 0.22 & $<0.001$ & 0.18 & 0.08 to 0.29 & 0.001 \\
\hline CRP & -1.57 & -3.91 to 0.71 & 0.182 & 0.85 & -1.39 to 3.09 & 0.447 \\
\hline FEV1 & 0.63 & 0.28 to 0.97 & 0.001 & 0.17 & -0.31 to 0.66 & 0.473 \\
\hline BMI & 1.56 & -1.19 to 4.31 & 0.258 & -2.91 & -5.67 to -0.15 & 0.039 \\
\hline Colonization & -13.84 & -29.76 to 2.08 & 0.087 & -5.51 & -21.74 to 10.73 & 0.496 \\
\hline FACED & -6.42 & -9.73 to -3.10 & $<0.001$ & -1.92 & -8.23 to 4.39 & 0.541 \\
\hline BSI & -2.83 & -4.13 to -1.53 & $<0.001$ & -0.24 & -2.73 to 2.26 & 0.848 \\
\hline
\end{tabular}

\section{TABLE 4: Factors affecting QOL physical}

MMRC: Modified Medical Research Council, CRP: C-reactive protein, BMI: body mass index, BSI: bronchiectasis severity index, CI: confidence interval; FACED: FEV1, age, colonization, extension, dyspnea; QOL: quality of life

In the role scale of QOL-B, multivariate regression analysis for predictors of low score showed association with 6MWD. There were no associations with MMRC, CRP, FEV1, BMI, colonizations, FACED scores, and BSI scores (Table 5). 


\section{Cureus}

\begin{tabular}{|c|c|c|c|c|c|c|}
\hline & \multicolumn{3}{|c|}{ Simple linear regression } & \multicolumn{3}{|c|}{ Multiple linear regression } \\
\hline & $\beta$ coefficient & $95 \% \mathrm{Cl}$ & p-Value & $\beta$ coefficient & $95 \% \mathrm{Cl}$ & $\mathrm{p}$-Value \\
\hline MMRC & -7.70 & -13.48 to -1.92 & 0.010 & 7.09 & -2.69 to 16.88 & 0.150 \\
\hline 6-minute walk test & 0.11 & 0.06 to 0.17 & $<0.001$ & 0.12 & 0.01 to 0.23 & 0.028 \\
\hline CRP & -0.89 & -2.88 to 1.09 & 0.369 & 0.97 & -1.24 to 3.17 & 0.379 \\
\hline FEV1 & 0.45 & 0.15 to 0.75 & 0.005 & 0.17 & -0.31 to 0.65 & 0.479 \\
\hline BMI & 1.85 & -0.46 to 4.12 & 0.108 & -1.24 & -3.95 to 1.48 & 0.361 \\
\hline Colonization & -4.77 & -0.18 to 9.02 & 0.489 & 4.48 & -11.46 to 20.42 & 0.572 \\
\hline FACED & -4.66 & -7.58 to -1.75 & 0.002 & -0.80 & -7.01 to 5.40 & 0.794 \\
\hline BSI & -2.16 & -3.29 to -1.02 & $<0.001$ & -1.74 & -4.20 to 0.71 & 0.157 \\
\hline
\end{tabular}

\section{TABLE 5: Factor affecting QOL role}

MMRC: Modified Medical Research Council, CRP: C-reactive protein, BMI: body mass index, BSI: bronchiectasis severity index, Cl: confidence interval; FACED: FEV1, age, colonization, extension, dyspnea; QOL: quality of life

In the vitality scale of QOL-B, multivariate regression analysis for predictors of low score showed associations with 6MWD. There were no associations with MMRC, CRP, FEV1, BMI, colonizations, FACED scores, and BSI scores (Table 6).

\begin{tabular}{|c|c|c|c|c|c|c|}
\hline & \multicolumn{3}{|c|}{ Simple linear regression } & \multicolumn{3}{|c|}{ Multiple linear regression } \\
\hline & $\beta$ coefficient & $95 \% \mathrm{Cl}$ & p-Value & $\beta$ coefficient & $95 \% \mathrm{Cl}$ & p-Value \\
\hline MMRC & -9.97 & -15.34 to -4.59 & 0.097 & 4.05 & -4.88 to 12.99 & 0.364 \\
\hline 6-minute walk test & 0.14 & 0.09 to 0.19 & $<0.001$ & 0.17 & 0.07 to 0.26 & 0.001 \\
\hline CRP & -1.51 & -3.44 to 0.43 & 0.125 & 0.86 & -1.15 to 2.87 & 0.391 \\
\hline FEV1 & 0.43 & 0.13 to 0.73 & 0.006 & 0.02 & -0.42 to 0.46 & 0.923 \\
\hline BMI & 2.45 & 0.25 to 4.65 & 0.30 & -0.87 & -3.34 to 1.61 & 0.483 \\
\hline Colonization & -11.21 & -24.54 to 2.11 & 0.097 & -6.09 & -20.66 to 8.47 & 0.401 \\
\hline FACED & -4.99 & -7.82 to -2.15 & 0.001 & -2.19 & -7.86 to 3.47 & 0.437 \\
\hline BSI & -2.23 & -3.33 to -1.11 & $<0.001$ & 0.05 & -2.19 to 2.29 & 0.967 \\
\hline
\end{tabular}

\section{TABLE 6: Factor affecting QOL vitality}

MMRC: Modified Medical Research Council, CRP: C-reactive protein, BMI: body mass index, BSI: bronchiectasis severity index, Cl: confidence interval; FACED: FEV1, age, colonization, extension, dyspnea; QOL: quality of life

In the emotional scale of QOL-B, multivariate regression analysis for predictors of low score showed association with 6MWD. There were no associations with MMRC, CRP, FEV1, BMI, colonizations, FACED scores, and BSI scores (Table 7). 


\section{Cureus}

\begin{tabular}{|c|c|c|c|c|c|c|}
\hline & \multicolumn{3}{|c|}{ Simple linear regression } & \multicolumn{3}{|c|}{ Multiple linear regression } \\
\hline & $\beta$ coefficient & $95 \% \mathrm{Cl}$ & p-Value & $\beta$ coefficient & $95 \% \mathrm{Cl}$ & $\mathrm{p}$-Value \\
\hline MMRC & -9.49 & -15.06 to -3.92 & 0.001 & -1.09 & -10.80 to 8.62 & 0.821 \\
\hline 6-minute walk test & 0.13 & 0.07 to 0.18 & $<0.001$ & 0.96 & -0.01 to 0.20 & 0.072 \\
\hline CRP & -2.56 & -4.42 to -0.70 & 0.008 & -1.12 & -3.30 to 1.07 & 0.306 \\
\hline FEV1 & 0.28 & -0.04 to 0.61 & 0.084 & -0.31 & -0.78 to 0.17 & 0.200 \\
\hline BMI & 2.92 & 0.73 to 5.09 & 0.010 & -0.15 & -2.84 to 2.54 & 0.911 \\
\hline Colonization & -10.19 & -23.79 to 3.40 & 0.138 & 2.96 & -12.86 to 18.79 & 0.706 \\
\hline FACED & -4.45 & -7.42 to -1.48 & 0.004 & -250 & -8.66 to 3.66 & 0.415 \\
\hline BSI & -2.18 & -3.32 to -1.03 & $<0.001$ & -0.47 & -2.90 to 1.97 & 0.700 \\
\hline
\end{tabular}

\section{TABLE 7: Factor affecting QOL emotional}

MMRC: Modified Medical Research Council, CRP: C-reactive protein, BMI: body mass index, BSI: bronchiectasis severity index, Cl: confidence interval; FACED: FEV1, age, colonization, extension, dyspnea; QOL: quality of life

In the social scale of QOL-B, multivariate regression analysis for predictors of low score showed that there were no associations with 6MWD, MMRC, CRP, FEV1, BMI, colonizations, FACED scores, or BSI scores (Table 8).

\begin{tabular}{|c|c|c|c|c|c|c|}
\hline & \multicolumn{3}{|c|}{ Simple linear regression } & \multicolumn{3}{|c|}{ Multiple linear regression } \\
\hline & $\beta$ coefficient & $95 \% \mathrm{Cl}$ & p-Value & $\beta$ coefficient & $95 \% \mathrm{Cl}$ & p-Value \\
\hline MMRC & -7.32 & -13.65 to -0.99 & 0.024 & 2.57 & -9.37 to 14.50 & 0.665 \\
\hline 6-minute walk test & 0.09 & 0.02 to 0.15 & 0.011 & 0.11 & -0.02 to 0.24 & 0.103 \\
\hline CRP & -0.77 & -2.91 to 1.37 & 0.472 & 0.45 & $-2.23-3.13$ & 0.735 \\
\hline FEV1 & 0.36 & 0.02 to 0.69 & 0.039 & 0.21 & -0.38 to 0.79 & 0.474 \\
\hline BMI & 0.23 & -2.29 to 2.74 & 0.857 & -2.44 & -5.75 to 0.86 & 0.142 \\
\hline Colonization & -7.67 & -22.39 to 7.04 & 0.299 & -2.56 & -22.01 to 16.88 & 0.791 \\
\hline FACED & -3.20 & -6.56 to 0.15 & 0.061 & 0.32 & -7.24 to 7.88 & 0.932 \\
\hline BSI & -1.57 & -2.90 to -0.24 & 0.022 & -0.73 & -3.72 to 2.26 & 0.623 \\
\hline
\end{tabular}

\section{TABLE 8: Factor affecting QOL social}

MMRC: Modified Medical Research Council, CRP: C-reactive protein, BMI: body mass index, BSI: bronchiectasis severity index, Cl: confidence interval; FACED: FEV1, age, colonization, extension, dyspnea; QOL: quality of life

In the treatment burden scale of QOL-B, multivariate regression analysis for predictors of low score showed associations with 6MWD and FEV1. There were no associations with MMRC, CRP, BMI, colonizations, FACED scores, and BSI scores (Table 9). 


\section{Cureus}

\begin{tabular}{|c|c|c|c|c|c|c|}
\hline & \multicolumn{3}{|c|}{ Simple linear regression } & \multicolumn{3}{|c|}{ Multiple linear regression } \\
\hline & $\beta$ coefficient & $95 \% \mathrm{Cl}$ & p-Value & $\beta$ coefficient & $95 \% \mathrm{Cl}$ & p-value \\
\hline MMRC & 3.13 & -4.46 to 10.73 & 0.408 & 7.98 & -5.81 to 21.77 & 0.246 \\
\hline 6-minute walk test & 0.02 & -0.05 to 0.09 & 0.548 & 0.16 & 0.03 to 0.29 & 0.017 \\
\hline CRP & -0.15 & -3.11 to 2.81 & 0.919 & -0.69 & -4.16 to 2.78 & 0.687 \\
\hline FEV1 & -0.43 & -0.79 to -0.07 & 0.021 & -0.74 & -1.38 to -0.11 & 0.023 \\
\hline BMI & -0.87 & -3.64 to 1.90 & 0.528 & -2.55 & -5.86 to 0.75 & 0.125 \\
\hline Colonization & -1.79 & -19.16 to 15.57 & 0.835 & -4.24 & -0.27 to 18.65 & 0.707 \\
\hline FACED & 2.08 & -1.73 to 5.90 & 0.275 & -2.92 & -10.73 to 4.89 & 0.451 \\
\hline BSI & 0.29 & -1.27 to 1.84 & 0.708 & 0.16 & -2.94 to 3.27 & 0.915 \\
\hline
\end{tabular}

\section{TABLE 9: Factor affecting QOL treatment burden}

MMRC: Modified Medical Research Council, CRP: C-reactive protein, BMI: body mass index, BSI: bronchiectasis severity index, Cl: confidence interval; FACED: FEV1, age, colonization, extension, dyspnea; QOL: quality of life

In the respiratory symptoms scale of QOL-B, multivariate regression analysis for predictors of low score showed association with 6MWD. There were no associations with MMRC, CRP, FEV1, BMI, colonizations, FACED scores, and BSI scores (Table 10).

\begin{tabular}{|c|c|c|c|c|c|c|}
\hline & \multicolumn{3}{|c|}{ Simple linear regression } & \multicolumn{3}{|c|}{ Multiple linear regression } \\
\hline & $\beta$ coefficient & $95 \% \mathrm{Cl}$ & $\mathrm{p}$-Value & $\beta$ coefficient & $95 \% \mathrm{Cl}$ & $p$-Value \\
\hline MMRC & -14.44 & -19.53 to -9.35 & $<0.001$ & -1.72 & -10.19 to 6.76 & 0.684 \\
\hline 6 -minute walk test & 0.17 & 0.12 to 0.22 & $<0.001$ & 0.11 & 0.02 to 0.20 & 0.018 \\
\hline CRP & -2.09 & -4.17 to -0.01 & 0.049 & 0.37 & -1.54 to 2.27 & 0.699 \\
\hline FEV1 & 0.62 & 0.31 to 0.92 & $<0.001$ & 0.15 & -0.27 to 0.56 & 0.481 \\
\hline BMI & 3.12 & 0.77 to 5.48 & 0.010 & -0.48 & -2.83 to 1.87 & 0.682 \\
\hline Colonization & -10.88 & -25.54 to 3.78 & 0.142 & 2.98 & -10.84 to 16.79 & 0.664 \\
\hline FACED & -6.39 & -9.31 to -3.47 & $<0.001$ & 0.91 & -4.48 to 6.29 & 0.733 \\
\hline BSI & -3.08 & -4.14 to -2.02 & $<0.001$ & -1.63 & -3.76 to 0.50 & 0.129 \\
\hline
\end{tabular}

\section{TABLE 10: Factor affecting QOL respiratory symptoms}

MMRC: Modified Medical Research Council, CRP: C-reactive protein, BMI: body mass index, BSI: bronchiectasis severity index, Cl: confidence interval; FACED: FEV1, age, colonization, extension, dyspnea; QOL: quality of life

In the health perceptions scale of QOL-B, multivariate regression analysis for predictors of low score showed association with 6MWD. There were no associations with MMRC, CRP, FEV1, BMI, colonizations, FACED scores, and BSI scores (Table 11). 


\begin{tabular}{|c|c|c|c|c|c|c|}
\hline & \multicolumn{3}{|c|}{ Simple linear regression } & \multicolumn{3}{|c|}{ Multiple linear regression } \\
\hline & $\beta$ coefficient & $95 \% \mathrm{Cl}$ & p-Value & $\beta$ coefficient & $95 \% \mathrm{Cl}$ & p-Value \\
\hline MMRC & -6.44 & -12.03 to -0.85 & 0.025 & 4.79 & -4.75 to 14.34 & 0.315 \\
\hline 6-minute walk test & 0.11 & 0.06 to 0.16 & $<0.001$ & 0.16 & 0.06 to 0.27 & 0.003 \\
\hline CRP & -1.55 & -3.39 to 0.29 & 0.097 & 0.04 & -2.10 to 2.19 & 0.967 \\
\hline FEV1 & 0.18 & -0.13 to 0.49 & 0.252 & -0.20 & -0.67 to 0.27 & 0.387 \\
\hline BMI & 1.71 & -0.45 to 3.87 & 0.118 & -1.29 & -3.93 to 1.36 & 0.330 \\
\hline Colonization & -9.46 & -22.29 to 3.36 & 0.144 & -4.64 & -20.19 to 10.91 & 0.549 \\
\hline FACED & -2.92 & -5.87 to 0.04 & 0.053 & -2.08 & -8.13 to 3.97 & 0.490 \\
\hline BSI & -1.58 & -2.73 to -0.42 & 0.009 & 0.05 & -2.34 to 2.45 & 0.964 \\
\hline
\end{tabular}

\section{TABLE 11: Factor affecting QOL health perceptions}

MMRC: Modified Medical Research Council, CRP: C-reactive protein, BMI: body mass index, BSI: bronchiectasis severity index, Cl: confidence interval; FACED: FEV1, age, colonization, extension, dyspnea; QOL: quality of life

\section{Discussion}

This study looked at the QOL-B scores and their various domains in patients with NCFB from a tertiary care center in South India. From an extensive review of the literature, no other similar studies could be identified and this is potentially the first such study from this geographical region. Our study is also unique in the fact that it included patients with very well-defined bronchiectasis who prospectively underwent multiple systems of scoring for the severity of NCFB including the BSI and FACED scores. They then prospectively were assessed for QOL-B scores, as well as outcome parameters including MMRC grading, BMI, 6MWD, CRP, and FEV1. This study demonstrated a significant impact on the QOL of NCFB patients based on the QOL-B scoring system. Almost all domains of QOL-B were found to be adversely impacted as measured by one or more of the outcome parameters but the FACED score, BSI score, 6MWD, and FEV1 showed associations across most scales while the other outcome parameters showed varying associations.

The 6MWD was found to correspond inversely with almost all domains of the QOL-B except for the treatment burden domain. 6MWD is a simple exercise test to evaluate the general functional responses of the pulmonary, cardiovascular, as well as muscular systems to assess daily physical activities and QOL of patients with NCFB. A study by Hsieh et al. reported that poorer 6MWD test results correlating with increased mortality in NCFB indicating that 6MWD may be an important predictor of QOL as well [6]. However, Jacques et al. reported otherwise in their study looking specifically at the role of 6MWD as an indicator of QOL in NCFB, stating that it was not a measure of QOL [7]. Given the highly ambiguous findings in previous literature, we believe that this study serves to add support to the 6MWD in the assessment of QOL in NCFB.

FEV1 predicted was also found to correspond inversely with almost all domains of the QOL-B except for the Health Perceptions domain. A large meta-analysis by Habib et al. reported that significant associations with FEV1\% predicted and QOL in NCFB [8]. These findings were corroborated in this study. The BSI score also showed a homogenous association across domains, with higher BSI grades corresponding to lower QOL domain scores. This was in keeping with previously reported data by McDonnell et al. [9]. The FACED score also showed similar homogenous association across QOL-B domains, with higher FACED grades corresponding to lower QOL domain scores, however, McDonnell et al. described various limitations in the use of FACED score in the assessment of QOL in NCFB [9]. The other parameters of QOL-B showed varying associations with the outcome parameters studied, with the heterogeneity of findings indicating limited clinical utility.

There were some limitations in this study. First, the number of patients enrolled in this study was limited, and all the patients were recruited from a single-center, which can limit the generalizability of the results of this study. Secondly, transversal variables related to NCFB, such as pulmonary hypertension or cardiovascular disorders and its impact on quality of life, were not included in the study due to the limited number of patients. Thirdly, evolutionary variables, including the number of exacerbations or hospitalizations due to NCFB or otherwise which could impact certain domains of QOL-B, were not included in the analysis. Finally, the impact of non-tuberculosis mycobacterial infection or colonization in these patients could not be studied. Hence, larger, multicenter studies may be needed with larger numbers of patients to corroborate our findings. 


\section{Conclusions}

The QOL is significantly reduced in NCFB and it may be quantified using the QOL-B questionnaire. The impact on QOL in NCFB may be assessed using validated tools such as the FACED and BSI scoring systems, as well as other well-established outcome parameters like 6MWD and FEV1 predicted. The role of outcome parameters such as BMI, CRP, MMRC, and colonization may be limited individually but may play a significant role when used in combinations.

\section{Additional Information}

\section{Disclosures}

Human subjects: Consent was obtained or waived by all participants in this study. Animal subjects: All authors have confirmed that this study did not involve animal subjects or tissue. Conflicts of interest: In compliance with the ICMJE uniform disclosure form, all authors declare the following: Payment/services info: All authors have declared that no financial support was received from any organization for the submitted work. Financial relationships: All authors have declared that they have no financial relationships at present or within the previous three years with any organizations that might have an interest in the submitted work. Other relationships: All authors have declared that there are no other relationships or activities that could appear to have influenced the submitted work.

\section{References}

1. Quint JK, Millett ER, Joshi M, et al.: Changes in the incidence, prevalence and mortality of bronchiectasis in the UK from 2004 to 2013: a population-based cohort study. Eur Respir J. 2016, 47:186-193. 10.1183/13993003.01033-2015

2. Smith MP: Diagnosis and management of bronchiectasis . CMAJ. 2017, 189:828-835. 10.1503/cmaj.160830

3. Schäfer J, Griese M, Chandrasekaran R, Chotirmall SH, Hartl D: Pathogenesis, imaging and clinical characteristics of CF and non-CF bronchiectasis. BMC Pulm Med. 2018, 18:79. 10.1186/s12890-018-0630-8

4. Quittner AL, O'Donnell AE, Salathe MA, et al.: Quality of Life Questionnaire-Bronchiectasis: final psychometric analyses and determination of minimal important difference scores. Thorax. 2015, 70:12-20. 10.1136/thoraxjnl-2014-205918

5. STROBE statement-checklist of items that should be included in reports of cross-sectional studies . (2007). Accessed: March 25, 2021: https://www.strobe-

statement.org/fileadmin/Strobe/uploads/checklists/STROBE_checklist_v4_cross-sectional.pdf.

6. Hsieh MH, Fang YF, Chung FT, et al.: Distance-saturation product of the 6-minute walk test predicts mortality of patients with non-cystic fibrosis bronchiectasis. J Thorac Dis. 2017, 9:3168-3176. $10.21037 /$ jtd.2017.08.53

7. Jacques PS, Gazzana MB, Palombini DV, Barreto SS, de Tarso Roth Dalcin P: Six-minute walk distance is not related to quality of life in patients with non-cystic fibrosis bronchiectasis. [Article in English, Portuguese]. J Bras Pneumol. 2012, 38:346-355. 10.1590/s1806-37132012000300010

8. Habib AR, Manji J, Wilcox PG, Javer AR, Buxton JA, Quon BS: A systematic review of factors associated with health-related quality of life in adolescents and adults with cystic fibrosis. Ann Am Thorac Soc. 2015, 12:420-428. 10.1513/AnnalsATS.201408-3930C

9. McDonnell MJ, Aliberti S, Goeminne PC, et al.: Multidimensional severity assessment in bronchiectasis: an analysis of seven European cohorts. Thorax. 2016, 71:1110-1118. 10.1136/thoraxjnl-2016-208481 